\title{
Classes of multivalent analytic and meromorphic functions with two fixed points
}

Jacek Dziok ${ }^{*}$

"Correspondence:

jdziok@univ.rzeszow.pl

Institute of Mathematics, University

of Rzeszów, Rzeszów, 35-310, Poland

\section{Abstract}

The object of the present paper is to investigate the coefficients estimates, distortion properties, the radii of starlikeness and convexity, subordination theorems, partial sums and integral mean inequalities for classes of functions with two fixed points. Some remarks depicting consequences of the main results are also mentioned. MSC: $30 \mathrm{C} 45 ; 30 C 50 ; 30 C 55$

Keywords: analytic functions; varying arguments; fixed points; Montel's normalization; subordination; Hadamard product

\section{Introduction}

Let $\mathcal{M}$ denote the class of functions which are holomorphic in $\mathcal{D}=\mathcal{D}(1)$, where

$$
\mathcal{D}(r)=\{z \in \mathbb{C}: 0<|z|<r\} .
$$

By $\mathcal{M}(p, k)$, where $p, k$ are integer, $p<k$, we denote the class of functions $f \in \mathcal{M}$ of the form

$$
f(z)=a_{p} z^{p}+\sum_{n=k}^{\infty} a_{n} z^{n} \quad\left(z \in \mathcal{D} ; a_{p}>0\right) .
$$

We note that for $p<0$ we have the class of functions which are meromorphic in $\mathcal{U}:=\mathcal{U}_{1}$, $\mathcal{U}_{r}:=\mathcal{D}_{r} \cup\{0\}$, and for $p \geq 0$ we obtain the class of functions which are analytic in $\mathcal{U}$.

Let $p>0, \alpha \in\langle 0, p), r \in(0,1\rangle$. A function $f \in \mathcal{M}(p, k)$ is said to be convex of order $\alpha$ in $\mathcal{D}(r)$ if

$$
\Re\left(1+\frac{z f^{\prime \prime}(z)}{f^{\prime}(z)}\right)>\alpha \quad\left(z \in \mathcal{D}(r) \backslash\left(f^{\prime}\right)^{-1}(\{0\})\right) .
$$

A function $f \in \mathcal{M}(p, k)$ is said to be starlike of order $\alpha$ in $\mathcal{D}(r)$ if

$$
\Re\left(\frac{z f^{\prime}(z)}{f(z)}\right)>\alpha \quad\left(z \in \mathcal{D}(r) \backslash f^{-1}(\{0\})\right) .
$$

We denote by $\mathcal{S}_{p}^{c}(\alpha)$ the class of all functions $f \in \mathcal{M}(p, p+1)$, which are convex of order $\alpha$ in $\mathcal{D}$ and by $\mathcal{S}_{p}^{*}(\alpha)$ we denote the class of all functions $f \in \mathcal{M}(p, p+1)$, which are starlike of order $\alpha$ in $\mathcal{D}$.

() 2013 Dziok: licensee Springer. This is an Open Access article distributed under the terms of the Creative Commons Attribution License (http://creativecommons.org/licenses/by/2.0), which permits unrestricted use, distribution, and reproduction in any medium, provided the original work is properly cited. 
Let $\mathcal{B} \subset \mathcal{M}(p, k), p>0$. We define the radius of starlikeness of order $\alpha$ and the radius of convexity of order $\alpha$ for the class $\mathcal{B}$ by

$$
\begin{aligned}
& R_{\alpha}^{*}(\mathcal{B}):=\inf _{f \in \mathcal{B}}(\sup \{r \in(0,1]: f \text { is starlike of order } \alpha \text { in } \mathcal{D}(r)\}), \\
& R_{\alpha}^{c}(\mathcal{B}):=\inf _{f \in \mathcal{B}}(\sup \{r \in(0,1]: f \text { is convex of order } \alpha \text { in } \mathcal{D}(r)\}),
\end{aligned}
$$

respectively.

We say that a function $f: \mathcal{U} \rightarrow \mathbb{C}$ is subordinate to a function $F: \mathcal{U} \rightarrow \mathbb{C}$, and write $f(z) \prec F(z)$ (or simply $f \prec F$ ), if there exists a function $\omega \in \mathcal{M}(\omega(0)=0,|\omega(z)|<1, z \in \mathcal{U})$, such that

$$
f(z)=F(\omega(z)) \quad(z \in \mathcal{U}) .
$$

In particular, if $F$ is univalent in $\mathcal{U}$, we have the following equivalence:

$$
f(z) \prec F(z) \Longleftrightarrow[f(0)=F(0) \wedge f(\mathcal{U}) \subset F(\mathcal{U})] .
$$

For functions $f, g \in \mathcal{M}$ of the form

$$
f(z)=\sum_{n=0}^{\infty} a_{n} z^{n}, \quad g(z)=\sum_{n=0}^{\infty} b_{n} z^{n} \quad(z \in \mathcal{D})
$$

by $f * g$ we denote the Hadamard product (or convolution) of $f$ and $g$, defined by

$$
(f * g)(z)=\sum_{n=0}^{\infty} a_{n} b_{n} z^{n} \quad(z \in \mathcal{D})
$$

For multivalent function $f \in \mathcal{M}(p, k)$, the normalization

$$
\left.z^{1-p} f(z)\right|_{z=0}=0 \quad \text { and }\left.\quad z^{-p} f(z)\right|_{z=0}=1
$$

is classical. One can obtain interesting results by applying Montel's normalization (cf. [1]) of the form

$$
\left.z^{1-p} f(z)\right|_{z=0}=0 \quad \text { and }\left.\quad z^{-p} f(z)\right|_{z=\rho}=1 \quad\left(\rho=|\rho| e^{i \eta}\right),
$$

where $\rho$ is a fixed point from the unit $\operatorname{disk} \mathcal{U}$. We see that for $\rho=0$ the normalization (4) is the classical normalization (3).

Let us denote by $\mathcal{M}_{\rho}(p, k)$ the class of functions $f \in \mathcal{M}(p, k)$ with Montel's normalization (4). It will be called the class of functions with two fixed points.

Also, by $\mathcal{T}^{\eta}(p, k), \eta \in \mathbb{R}$, we denote the class of functions $f \in \mathcal{M}(p, k)$ of the form

$$
f(z)=a_{p} z^{p}-\sum_{n=k}^{\infty}\left|a_{n}\right| e^{-(n+p) \eta} z^{n} \quad(z \in \mathcal{D}) .
$$


In particular, we obtain the class $\mathcal{T}^{0}(p, k)$ of functions with negative coefficients. Moreover, we define

$$
\mathcal{T}(p, k):=\bigcup_{\eta \in \mathbb{R}} \mathcal{T}^{\eta}(p, k)
$$

The classes $\mathcal{T}(p, k)$ and $\mathcal{T}^{\eta}(p, k)$ are called the classes of functions with varying argument of coefficients. The class $\mathcal{T}(1,2)$ was introduced by Silverman [2] (see also [3]). It is easy to show that for $f \in \mathcal{T}(p, k), p>0$, the condition (2) is equivalent to the following:

$$
\left|\frac{z f^{\prime}(z)}{f(z)}-p\right|<p-\alpha \quad\left(z \in \mathcal{D}(r) \backslash f^{-1}(\{0\})\right) .
$$

Let $A, B, \delta$ be real parameters, $\delta \geq 0,0 \leq B \leq 1,-1 \leq A<B$, and let $\varphi, \phi \in \mathcal{M}(p, k)$. By $\mathcal{W}=\mathcal{W}(p, k ; \phi, \varphi ; A, B ; \delta)$ we denote the class of functions $f \in \mathcal{M}(p, k)$ such that

$$
(\varphi * f)(z) \neq 0 \quad(z \in \mathcal{D})
$$

and

$$
\frac{(\phi * f)(z)}{(\varphi * f)(z)}-\delta\left|\frac{(\phi * f)(z)}{(\varphi * f)(z)}-1\right| \prec \frac{1+A z}{1+B z}
$$

If $0<B<1$, then the function

$$
h(z)=\frac{1+A z}{1+B z} \quad(z \in \mathcal{D})
$$

is univalent in $\mathcal{U}$ and maps $\mathcal{U}$ onto the $\operatorname{disk}\{w \in \mathbb{C}:|w-a|<R\}$, where

$$
a=\frac{1-A B}{1-B^{2}}, \quad R=\frac{B-A}{1-B^{2}} .
$$

Thus, by definition of subordination the condition (9) is equivalent to the following:

$$
\left|\frac{(\phi * f)(z)}{(\varphi * f)(z)}-\delta\right| \frac{(\phi * f)(z)}{(\varphi * f)(z)}-1\left|-\frac{1-A B}{1-B^{2}}\right|<\frac{B-A}{1-B^{2}} \quad(z \in \mathcal{D}) .
$$

If $B=1$, then the function (10) maps the disc $\mathcal{D}$ onto the half-plane $\left\{w \in \mathbb{C}: \Re[w]>\frac{1+A}{2}\right\}$. Thus, the condition (9) is equivalent to the following:

$$
\delta\left|\frac{(\phi * f)(z)}{(\varphi * f)(z)}-1\right|-\Re\left\{\frac{(\phi * f)(z)}{(\varphi * f)(z)}-1\right\}<\frac{1-A}{2} \quad(z \in \mathcal{D}) .
$$

Now, we define the classes of functions with varying argument of coefficients related to the class $\mathcal{W}=\mathcal{W}(p, k ; \phi, \varphi ; A, B ; \delta)$. Let us denote

$$
\begin{aligned}
& \mathcal{W}_{\rho}=\mathcal{W}_{\rho}(p, k ; \phi, \varphi ; A, B ; \delta):=\mathcal{A}_{\rho}(p, k) \cap \mathcal{W}(p, k ; \phi, \varphi ; A, B ; \delta), \\
& \mathcal{T} \mathcal{W}^{\eta}=\mathcal{T} \mathcal{W}^{\eta}(p, k ; \phi, \varphi ; A, B ; \delta):=\mathcal{T}^{\eta}(p, k) \cap \mathcal{W}(p, k ; \phi, \varphi ; A, B ; \delta),
\end{aligned}
$$




$$
\begin{aligned}
& \mathcal{T} \mathcal{W}_{\rho}^{\eta}=\mathcal{T} \mathcal{W}_{\rho}^{\eta}(p, k ; \phi, \varphi ; A, B ; \delta):=\mathcal{M}_{\rho}(p, k) \cap \mathcal{T} \mathcal{W}^{\eta}(p, k ; \phi, \varphi ; A, B ; \delta), \\
& \mathcal{T} \mathcal{W}_{\rho}=\mathcal{T} \mathcal{W}_{\rho}(p, k ; \phi, \varphi ; A, B ; \delta):=\mathcal{T}(p, k) \cap \mathcal{W}_{\rho}(p, k ; \phi, \varphi ; A, B ; \delta) .
\end{aligned}
$$

The class $\mathcal{W}=\mathcal{W}(p, k ; \phi, \varphi ; A, B ; \delta)$ unifies various new and also well-known classes of analytic or meromorphic functions; see for example [1-36].

For the presented investigations we assume that $\varphi, \phi$ are the functions of the form

$$
\begin{aligned}
& \varphi(z)=z^{p}+\sum_{n=k}^{\infty} \alpha_{n} z^{n}, \quad \phi(z)=z^{p}+\sum_{n=k}^{\infty} \beta_{n} z^{n} \quad(z \in \mathcal{D}), \\
& 0 \leq \alpha_{n}<\beta_{n}\left(n \in \mathbb{N}_{k}:=\{k, k+1, \ldots\}\right) .
\end{aligned}
$$

Moreover, let us put

$$
d_{n}:=(\delta+1)(1+B) \beta_{n}-(\delta(B+1)+A+1) \alpha_{n} \quad\left(n \in \mathbb{N}_{k}\right)
$$

The object of the present paper is to investigate the coefficients estimates, distortion properties, the radii of starlikeness and convexity, subordination theorems, partial sums and integral mean inequalities for the classes of functions with varying argument of coefficients. Some remarks depicting consequences of the main results are also mentioned.

\section{Coefficients estimates}

We first mention a sufficient condition for the function to belong to the class $\mathcal{W}$.

Theorem 1 Let $0 \leq B \leq 1$ and $-1 \leq A<B$. If $\in \mathcal{M}_{\rho}(p, k)$ and

$$
\sum_{n=k}^{\infty} d_{n}\left|a_{n}\right| \leq(B-A) a_{p},
$$

then $f \in \mathcal{W}$.

Proof If $0 \leq B<1$, then we have

$$
\begin{aligned}
& \left|\frac{(\phi * f)(z)}{(\varphi * f)(z)}-\delta\right| \frac{(\phi * f)(z)}{(\varphi * f)(z)}-1\left|-\frac{1-A B}{1-B^{2}}\right| \\
& \quad \leq(\delta+1)\left|\frac{(\phi * f)(z)}{(\varphi * f)(z)}-1\right|+\frac{B(B-A)}{1-B^{2}} \\
& \quad \leq(\delta+1) \frac{\sum_{n=k}^{\infty}\left(\beta_{n}-\alpha_{n}\right)\left|a_{n}\right||z|^{n-p}}{a_{p}-\sum_{n=k}^{\infty} \alpha_{n}\left|a_{n}\right||z|^{n-p}}+\frac{B(B-A)}{1-B^{2}} .
\end{aligned}
$$

Thus, by (15), we obtain (11) and consequently $f \in \mathcal{W}$. Let now $B=1$. Then simply calculations give

$$
\begin{aligned}
& \delta\left|\frac{(\phi * f)(z)}{(\varphi * f)(z)}-1\right|-\mathfrak{R}\left\{\frac{(\phi * f)(z)}{(\varphi * f)(z)}-1\right\} \\
& \quad \leq(\delta+1)\left|\frac{(\phi * f)(z)}{(\varphi * f)(z)}-1\right| \leq(\delta+1) \frac{\sum_{n=k}^{\infty}\left(\beta_{n}-\alpha_{n}\right)\left|a_{n}\right||z|^{n-p}}{a_{p}-\sum_{n=k}^{\infty} \alpha_{n}\left|a_{n}\right||z|^{n-p}} .
\end{aligned}
$$

Thus, by (15) we obtain (12). Hence $f \in \mathcal{W}$ and the proof is complete. 
Theorem 2 Let $f \in \mathcal{T}^{\eta}(p, k)$. Then $f \in \mathcal{T} \mathcal{W}^{\eta}$ if and only if the condition (15) holds true.

Proof Let $f \in \mathcal{T} \mathcal{W}^{\eta}$. In view of Theorem 1, we need only show that $f$ satisfies the coefficient inequality (15). Putting $z=r e^{i \eta}$ in the conditions (11) and (12) we obtain

$$
(\delta+1) \frac{\sum_{n=2}^{\infty}\left(\beta_{n}-\alpha_{n}\right)\left|a_{n}\right| r^{n-p}}{a_{p}-\sum_{n=2}^{\infty} \alpha_{n}\left|a_{n}\right| r^{n-p}}<\frac{B-A}{1+B} .
$$

By (8), it is clear that the denominator of the left hand side cannot vanish for $r \in\langle 0,1)$. Moreover, it is positive for $r=0$, and in consequence for $r \in\langle 0,1)$. Thus, we have

$$
\sum_{n=2}^{\infty} d_{n}\left|a_{n}\right| r^{n-p}<(B-A) a_{p}
$$

which, upon letting $r \rightarrow 1^{-}$, readily yields the assertion (15).

By applying Theorem 2, we can deduce following result.

Theorem 3 Let $f \in \mathcal{T}^{\eta}(p, k)$. Then $f \in \mathcal{T} \mathcal{W}_{\rho}^{\eta}$ if and only if it satisfies (4) and

$$
\sum_{n=k}^{\infty}\left(d_{n}-(B-A)|\rho|^{n-p}\right)\left|a_{n}\right| \leq B-A .
$$

Proof For a function $f \in \mathcal{T}^{\eta}(p, k)$ with the normalization (4), we have

$$
a_{p}=1+\sum_{n=k}^{\infty}\left|a_{n}\right||\rho|^{n-p}
$$

Then the conditions (15) and (16) are equivalent.

From Theorem 3, we obtain the following lemma.

Lemma 1 Let there exist an integer $n_{0} \in \mathbb{N}_{k}$ such that

$$
d_{n_{0}}-(B-A)|\rho|^{n_{0}-p} \leqq 0
$$

Then the function

$$
f_{n_{0}}(z)=\left(1+a \rho^{n_{0}-p}\right) z^{p}-a e^{i\left(p-n_{0}\right) \eta} z^{n_{0}}
$$

belongs to the class $\mathcal{T} \mathcal{W}_{\rho}^{\eta}$ for all positive real numbers a. Moreover, for all $n \in \mathbb{N}_{k}$ such that

$$
d_{n}-(B-A)|\rho|^{n-p}>0 \text {, }
$$

the functions

$$
f_{n}(z)=\left(1+a \rho^{n_{0}-p}+b z^{n-p}\right) z^{p}-a e^{i\left(p-n_{0}\right) \eta} z^{n_{0}}-b e^{i(p-n) \eta} z^{n},
$$


belongs to the class $\mathcal{T} \mathcal{W}_{\rho}^{\eta}$ for all positive real numbers $a$ and

$$
b=\frac{B-A+\left((B-A)|\rho|^{n_{0}-p}-d_{n_{0}}\right) a}{d_{n}-(B-A)|\rho|^{n-p}} .
$$

By Lemma 1 and Theorem 3, we have following two corollaries.

\section{Corollary 1 Let}

$$
d_{n}-(B-A)|\rho|^{n-p} \geq 0 \quad\left(n \in \mathbb{N}_{k}\right) .
$$

If

$$
d_{n}-(B-A)|\rho|^{n-p}>0
$$

then the nth coefficient of the class $\mathcal{T} \mathcal{W}_{\rho}^{\eta}$ satisfies the following inequality:

$$
\left|a_{n}\right| \leqq \frac{B-A}{d_{n}-(B-A)|\rho|^{n-p}}
$$

The estimation (20) is sharp, the function $f_{n, \eta}$ of the form

$$
f_{n, \eta}(z)=\frac{d_{n} z^{p}-(B-A) e^{i(p-n) \eta} z^{n}}{d_{n}-(B-A)|\rho|^{n-p}} \quad(z \in \mathcal{D})
$$

is the extremal function.

\section{Corollary 2 If}

$$
d_{n}-(B-A)|\rho|^{n-p}=0,
$$

then the nth coefficient of the class $\mathcal{T} \mathcal{W}_{\rho}^{\eta}$ is unbounded. Moreover, if there exists $n_{0} \in \mathbb{N}_{k}$ such that

$$
d_{n_{0}}-(B-A)|\rho|^{n_{0}-p}<0,
$$

then all of the coefficients of the class $\mathcal{T} \mathcal{W}_{\rho}^{\eta}$ are unbounded.

By putting $\rho=0$ in Theorem 3 and Corollary 1, we have the corollaries listed below.

Corollary 3 Let $f \in \mathcal{T}^{\eta}(p, k)$. Then $f \in \mathcal{T} \mathcal{W}_{0}^{\eta}$ if and only if

$$
\sum_{n=k}^{\infty} d_{n}\left|a_{n}\right| \leq B-A
$$

Corollary 4 If $\in \mathcal{T} \mathcal{W}_{0}^{\eta}$, then

$$
a_{n} \leq \frac{B-A}{d_{n}} \quad\left(n \in \mathbb{N}_{k}\right)
$$


The result is sharp. The functions $f_{n, \eta}$ of the form

$$
f_{n, \eta}(z)=z^{p}-\frac{B-A}{d_{n}} e^{i(p-n) \eta} z^{n} \quad\left(z \in \mathcal{D} ; n \in \mathbb{N}_{k}\right)
$$

are the extremal functions.

\section{Distortion theorems}

From Theorem 2, we have the following lemma.

Lemma 2 Let $f \in \mathcal{T} \mathcal{W}_{\rho}^{\eta}$. If the sequence $\left\{d_{n}\right\}$ satisfies the inequality

$$
0<d_{k}-(B-A)|\rho|^{k-p} \leq d_{n}-(B-A)|\rho|^{n-p} \quad\left(n \in \mathbb{N}_{k}\right)
$$

then

$$
\sum_{n=k}^{\infty}\left|a_{n}\right| \leq \frac{B-A}{d_{k}-(B-A)|\rho|^{k-p}}
$$

Moreover, if

$$
0<\frac{d_{k}-(B-A)|\rho|^{k-p}}{k} \leq \frac{d_{n}-(B-A)|\rho|^{n-p}}{n} \quad\left(n \in \mathbb{N}_{k}\right),
$$

then

$$
\sum_{n=k}^{\infty} n\left|a_{n}\right| \leq \frac{k(B-A)}{d_{k}-(B-A)|\rho|^{k-p}} .
$$

The second part of Lemma 2 may be formulated in terms of $\sigma$-neighborhood $N_{\sigma}$ defined by

$$
N_{\sigma}=\left\{f(z)=a_{p} z^{p}+\sum_{n=k}^{\infty} a_{n} z^{n} \in \mathcal{T}^{\eta}(p, k): \sum_{n=k}^{\infty} n\left|a_{n}\right| \leq \sigma\right\}
$$

as the following corollary.

Corollary 5 If the sequence $\left\{d_{n}\right\}$ satisfies (26), then $\mathcal{T} \mathcal{W}_{\rho}^{\eta} \subset N_{\sigma}$, where

$$
\delta=\frac{k(B-A)}{d_{k}-(B-A)|\rho|^{k-p}} .
$$

Theorem 4 Let $f \in \mathcal{T} \mathcal{W}_{\rho}^{\eta},|z|=r<1$. If the sequence $\left\{d_{n}\right\}$ satisfies (25), then

$$
\phi(r) \leq|f(z)| \leq \frac{d_{k} r^{p}+(B-A) r^{k}}{d_{k}-(B-A)|\rho|^{k-p}}
$$

where

$$
\phi(r):= \begin{cases}r^{p} & (r \leq \rho), \\ \frac{d_{k} r^{p}-(B-A) r^{k}}{d_{k}-(B-A)|\rho|^{k-p}} & (r>\rho) .\end{cases}
$$


Moreover, if (26) holds, then

$$
p a_{p} r^{p-1}-\frac{k(B-A)}{d_{k}-(B-A)|\rho|^{k-p}} r^{k-1} \leq\left|f^{\prime}(z)\right| \leq \frac{p d_{k} r^{p}+k(B-A) r^{k-1}}{d_{k}-(B-A)|\rho|^{k-p}} .
$$

The result is sharp, with the extremal function $f_{k, \eta}$ of the form $(21)$ and $f_{0}(z)=z$.

Proof Suppose that the function $f$ of the form (1) belongs to the class $\mathcal{T} \mathcal{W}_{\rho}^{\eta}$. By Lemma 2 we have

$$
\begin{aligned}
|f(z)| & =\left|a_{p} z^{p}+\sum_{n=k}^{\infty} a_{n} z^{n}\right| \leq r^{p}\left(a_{p}+\sum_{n=k}^{\infty}\left|a_{n}\right| r^{n-p}\right) \\
& \leq r^{p}\left(1+\sum_{n=k}^{\infty}\left|a_{n}\right||\rho|^{n-p}+\sum_{n=k}^{\infty}\left|a_{n}\right| r^{n-p}\right) \\
& \leq r^{p}\left(1+\left(|\rho|^{k-p}+r^{k-p}\right) \sum_{n=k}^{\infty}\left|a_{n}\right|\right) \leq \frac{d_{k} r^{p}+(B-A) r^{k}}{d_{k}-(B-A)|\rho|^{k-p}}
\end{aligned}
$$

and

$$
|f(z)| \geq r^{p}\left(a_{p}-\sum_{n=k}^{\infty}\left|a_{n}\right| r^{n-p}\right)=r^{p}\left(1+\sum_{n=k}^{\infty}\left(|\rho|^{n-p}-r^{n-p}\right)\left|a_{n}\right|\right) .
$$

If $r \leq \rho$, then we obtain $|f(z)| \geq r^{p}$. If $r>\rho$, then the sequence $\left\{\left(\rho^{n-p}-r^{n-p}\right)\right\}$ is decreasing and negative. Thus, by (30), we obtain

$$
|f(z)| \geq r^{p}\left(1-\left(r^{k-p}-|\rho|^{k-p}\right) \sum_{n=2}^{\infty} a_{n}\right) \geq \frac{d_{k} r^{p}-(B-A) r^{k}}{d_{k}-(B-A)|\rho|^{k-p}}
$$

and we have the assertion (27). Making use of Lemma 2, in conjunction with (17), we readily obtain the assertion (29) of Theorem 4.

Putting $\rho=0$ in Theorem 4 we have the following corollary.

Corollary 6 Let $f \in \mathcal{T} \mathcal{W}_{0}^{\eta},|z|=r<1$. If $d_{k} \leq d_{n}\left(n \in \mathbb{N}_{k}\right)$, then

$$
r^{p}-\frac{B-A}{d_{k}} r^{k} \leq|f(z)| \leq r^{p}+\frac{B-A}{d_{k}} r^{k} .
$$

Moreover, if $n d_{k} \leq k d_{n}\left(n \in \mathbb{N}_{k}\right)$, then

$$
p r^{p-1}-\frac{k(B-A)}{d_{k}} r^{k-1} \leq\left|f^{\prime}(z)\right| \leq p r^{p-1}+\frac{k(B-A)}{d_{k}} r^{k-1} .
$$

The result is sharp, with the extremal function $f_{k, \eta}$ of the form (24). 


\section{The radii of convexity and starlikeness}

Theorem 5 If $p>0$, then

$$
R_{\alpha}^{*}\left(\mathcal{T} \mathcal{W}^{\eta}\right)=\inf _{n \geq k}\left(\frac{(p-\alpha) d_{n}}{(n-\alpha)(B-A)}\right)^{\frac{1}{n-p}}
$$

The functions $f_{n, \eta}$ of the form

$$
f_{n, \eta}(z)=a_{p}\left(z^{p}-\frac{B-A}{d_{n}} e^{i(p-n) \eta} z^{n}\right) \quad\left(z \in \mathcal{U} ; n \in \mathbb{N}_{k} ; a_{p}>0\right)
$$

are the extremal functions.

Proof A function $f \in \mathcal{T}^{\eta}(p, k)$ of the form (1) is starlike of order $\alpha$ in $\mathcal{U}(r)$ if and only if it satisfies the condition (7). Since

$$
\left|\frac{z f^{\prime}(z)}{f(z)}-p\right|=\left|\frac{\sum_{n=k}^{\infty}(n-p) a_{n} z^{n}}{a_{p} z^{p}+\sum_{n=k}^{\infty} a_{n} z^{n}}\right| \leq \frac{\sum_{n=k}^{\infty}(n-p)\left|a_{n}\right||z|^{n-p}}{a_{p}-\sum_{n=k}^{\infty}\left|a_{n}\right||z|^{n-p}},
$$

the condition (7) is true if

$$
\sum_{n=k}^{\infty} \frac{n-\alpha}{p-\alpha}\left|a_{n}\right| r^{n-p} \leq a_{p}
$$

By Theorem 2, we have

$$
\sum_{n=k}^{\infty} \frac{d_{n}}{B-A}\left|a_{n}\right| \leq a_{p}
$$

Thus, the condition (34) is true if

$$
\frac{n-\alpha}{p-\alpha} r^{n-p} \leq \frac{d_{n}}{B-A} \quad\left(n \in \mathbb{N}_{k}\right)
$$

that is, if

$$
r \leq\left(\frac{(p-\alpha) d_{n}}{(n-\alpha)(B-A)}\right)^{\frac{1}{n-p}} \quad\left(n \in \mathbb{N}_{k}\right)
$$

It follows that each function $f \in \mathcal{T} \mathcal{W}^{\eta}$ is starlike of order $\alpha$ in $\mathcal{U}(r)$, where

$$
r=\inf _{n \geq k}\left(\frac{(p-\alpha) d_{n}}{(n-\alpha)(B-A)}\right)^{\frac{1}{n-p}} .
$$

The functions $f_{n, \eta}$ of the form (33) realize equality in (35), and the radius $r$ cannot be larger. Thus we have (32). 
The following result may be proved in much the same way as Theorem 5 .

Theorem 6 If $p>0$, then

$$
R_{\alpha}^{c}\left(\mathcal{T} \mathcal{W}^{\eta}\right)=\inf _{n \geq k}\left(\frac{(p-\alpha) d_{n}}{n(n-\alpha)(B-A)}\right)^{\frac{1}{n-p}}
$$

The functions $f_{n, \eta}$ of the form (33) are the extremal functions.

It is clear that for

$$
a_{p}=\frac{d_{n}}{d_{n}-(B-A)|\rho|^{n-p}}>0
$$

the extremal function $f_{n, \eta}$ of the form (33) belongs to the class $\mathcal{T} \mathcal{W}_{\rho}^{\eta}$. Moreover, we have

$$
\mathcal{T} \mathcal{W}_{\rho}^{\eta} \subset \mathcal{T} \mathcal{W}^{\eta}
$$

Thus, by Theorems 5 and 6 we have the following corollary.

Corollary 7 Let the sequence $\left\{d_{n}-(B-A)|\rho|^{n-p}\right\}$ be positive, $p>0$. Then

$$
\begin{aligned}
& R_{\alpha}^{*}\left(\mathcal{T} \mathcal{W}_{\rho}^{\eta}\right)=\inf _{n \geq k}\left(\frac{(p-\alpha) d_{n}}{(n-\alpha)(B-A)}\right)^{\frac{1}{n-p}}, \\
& R_{\alpha}^{c}\left(\mathcal{T} \mathcal{W}_{\rho}^{\eta}\right)=\inf _{n \geq k}\left(\frac{(p-\alpha) d_{n}}{n(n-\alpha)(B-A)}\right)^{\frac{1}{n-p}} .
\end{aligned}
$$

\section{Subordination results}

Before stating and proving our subordination theorems for the class $\mathcal{T} \mathcal{W}^{\eta}$, we need the following definition and lemma.

Definition 1 A sequence $\left\{b_{n}\right\}$ of complex numbers is said to be a subordinating factor sequence if for each function $f \in \mathcal{S}^{c}$ we have

$$
\sum_{n=1}^{\infty} b_{n} a_{n} z^{n} \prec f(z) \quad\left(a_{1}=1\right) .
$$

Lemma 3 [36] A sequence $\left\{b_{n}\right\}$ is a subordinating factor sequence if and only if

$$
\Re\left\{1+2 \sum_{n=1}^{\infty} b_{n} z^{n}\right\}>0 \quad(z \in \mathcal{D}) .
$$

Theorem 7 Let the sequence $\left\{d_{n}\right\}$ satisfy the inequality (25). If $g \in \mathcal{S}^{c}$ and $f \in \mathcal{T} \mathcal{W}^{\eta}$, then

$$
\left[\varepsilon z^{1-p} f(z)\right] * g(z) \prec g(z)
$$


and

$$
\Re\left[z^{1-p} f(z)\right]>-\frac{1}{2 \varepsilon} \quad(z \in \mathcal{D})
$$

where

$$
\varepsilon=\frac{d_{k}}{2 a_{p}\left(B-A+d_{k}\right)} .
$$

If $p$ and $(k-p)$ are odd, and $\eta=0$, then the constant factor $\varepsilon$ cannot be replaced by a larger number.

Proof Let a function $f$ of the form (1) belong to the class $\mathcal{T} \mathcal{W}^{\eta}$ and suppose that a function $g$ of the form

$$
g(z)=\sum_{n=1}^{\infty} c_{n} z^{n} \quad\left(c_{1}=1 ; z \in \mathcal{D}\right)
$$

belongs to the class $\mathcal{S}^{c}$. Then

$$
\left[\varepsilon z^{1-p} f(z)\right] * g(z)=\sum_{n=1}^{\infty} b_{n} c_{n} z^{n} \quad(z \in \mathcal{D})
$$

where

$$
b_{n}= \begin{cases}\varepsilon a_{p} & \text { if } n=1, \\ 0 & \text { if } 2 \leq n \leq k-p \\ \varepsilon a_{n+p-1} & \text { if } n>k-p .\end{cases}
$$

Thus, by Definition 1, the subordination result (39) holds true if $\left\{b_{n}\right\}$ is the subordinating factor sequence. By (25), we have

$$
\begin{aligned}
\Re\left\{1+2 \sum_{n=1}^{\infty} b_{n} z^{n}\right\} & =\Re\left\{1+2 \varepsilon a_{p} z+\sum_{n=k}^{\infty} \frac{d_{k}}{B-A+d_{k}} a_{n} z^{n-p}\right\} \\
& \geq 1-2 \varepsilon r-\frac{r}{\left(B-A+d_{k}\right) a_{p}} \sum_{n=k}^{\infty} d_{n}\left|a_{n}\right| \quad(|z|=r<1) .
\end{aligned}
$$

Thus, by using Theorem 2, we obtain

$$
\Re\left\{1+2 \sum_{n=1}^{\infty} b_{n} z^{n}\right\} \geq 1-\frac{d_{k}}{B-A+d_{k}} r-\frac{B-A}{B-A+d_{k}} r>0 .
$$

This evidently proves the inequality (38) and hence the subordination result (39). The inequality (40) follows from (39) by taking

$$
g(z)=\frac{z}{1-z}=\sum_{n=1}^{\infty} z^{n} \quad(z \in \mathcal{D})
$$


Next, we observe that the function $f_{k, \eta}$ of the form (33) belongs to the class $\mathcal{T} \mathcal{W}^{\eta}$. If $p$ and $(k-p)$ are odd, and $\eta=0$, then

$$
\left.z^{1-p} f_{k, \eta}(z)\right|_{z=-1}=-\frac{1}{2 \varepsilon}
$$

and the constant (41) cannot be replaced by any larger one.

Remark 1 By using (17) in Theorem 7, we obtain the result related to the class $\mathcal{T} \mathcal{W}_{\rho}^{\eta}$. Moreover, by putting $\rho=0$, we have the following corollary.

Corollary 8 Let the sequence $\left\{d_{n}\right\}$ satisfy the inequality (25). If $g \in \mathcal{S}^{c}$ and $f \in \mathcal{T} \mathcal{W}_{0}^{\eta}$, then conditions (39) and (40) hold true. If $p$ and $(k-p)$ are odd, and $\eta=0$, then the constant factor $\varepsilon=\frac{d_{k}}{2\left(B-A+d_{k}\right)}$ cannot be replaced by a larger number.

\section{Integral means inequalities}

Due to Littlewood [22], we obtain integral means inequalities for the functions from the class $\mathcal{T} \mathcal{W}^{\eta}$.

Lemma 4 [22] Let $f$, $g$ be functions analytic in $\mathcal{U}$. If $\prec$ g, then

$$
\int_{0}^{2 \pi}\left|f\left(r e^{i \theta}\right)\right|^{\lambda} d \theta \leq \int_{0}^{2 \pi}\left|g\left(r e^{i \theta}\right)\right|^{\lambda} d \theta \quad(0<r<1,0<\lambda) .
$$

Applying Lemma 4 and Theorem 2, we prove the following result.

Theorem 8 Let the sequence $\left\{d_{n}\right\}$ satisfy $(25), k=p+1$. Iff $\in \mathcal{T} \mathcal{W}_{\rho}$, then

$$
\int_{0}^{2 \pi}|f(z)|^{\lambda} d \theta \leq \int_{0}^{2 \pi}\left|f_{p+1, \eta}(z)\right|^{\lambda} d \theta \quad\left(0<r<1,0<\lambda ; z=r e^{i \theta}\right)
$$

where $f_{p+1, \eta}$ is defined by (33).

Proof For function $f$ of the form (1), the inequality (43) is equivalent to the following:

$$
\int_{0}^{2 \pi}\left|a_{p}+\sum_{n=p+1}^{\infty} a_{n} z^{n-p}\right|^{\lambda} d \theta \leq \int_{0}^{2 \pi}\left|a_{p}-\frac{B-A}{d_{p+1}} e^{-i \eta} z\right|^{\lambda} d \theta \quad\left(z=r e^{i \theta}\right) .
$$

By Lemma 4, it suffices to show that

$$
\sum_{n=p+1}^{\infty} a_{n} z^{n-p} \prec-\frac{B-A}{d_{p+1}} e^{-i \eta} z
$$

Setting

$$
w(z)=-\sum_{n=p+1}^{\infty} \frac{d_{p+1} e^{i \eta}}{B-A} a_{n} z^{n-p} \quad(z \in \mathcal{D})
$$


and using (25) and Theorem 2 we obtain

$$
|w(z)|=\left|\sum_{n=p+1}^{\infty} \frac{d_{p+1}}{B-A} a_{n} z^{n-p}\right| \leq|z| \sum_{n=p+1}^{\infty} \frac{d_{n}}{B-A}\left|a_{n}\right| \leq|z| \quad(z \in \mathcal{D})
$$

and

$$
\sum_{n=p+1}^{\infty} a_{n} z^{n-p}=-\frac{B-A}{d_{p+1}} e^{-i \eta} w(z) \quad(z \in \mathcal{D})
$$

Thus, by definition of subordination we have (44) and this completes the proof.

By using (17) in Theorem 8 we have the following corollary.

Corollary 9 Let the sequence $\left\{d_{n}\right\}$ satisfy (25), $k=p+1$. Iff $\in \mathcal{T} \mathcal{W}_{\rho}^{\eta}$, then

$$
\int_{0}^{2 \pi}\left|f\left(r e^{i \theta}\right)\right|^{\lambda} d \theta \leq \int_{0}^{2 \pi}\left|f_{p+1, \eta}\left(r e^{i \theta}\right)\right|^{\lambda} d \theta \quad\left(0<r<1, \lambda>0 ; z=r e^{i \theta}\right)
$$

where $f_{p+1, \eta}$ is defined by (21).

\section{Partial sums}

Let $f$ be a function of the form (1). Due to Silvia [27], we investigate the partial sums $f_{m}$ of the function $f$ defined by

$$
f_{k-1}(z)=a_{p} z^{p}, \quad f_{m}(z)=a_{p} z^{p}+\sum_{n=k}^{m} a_{n} z^{n} \quad\left(m \in \mathbb{N}_{k}\right)
$$

In this section, we consider partial sums of functions in the class $\mathcal{T} \mathcal{W}^{\eta}$ and obtain sharp lower bounds for the ratios of real part of $f$ to $f_{m}$ and $f^{\prime}$ to $f_{m}^{\prime}$.

Theorem 9 Let the sequence $\left\{d_{n}\right\}$ be increasing and $d_{k} \geq B-A$. Iff $\in \mathcal{T} \mathcal{W}^{\eta}$, then

$$
\operatorname{Re}\left\{\frac{f(z)}{f_{m}(z)}\right\} \geq 1-\frac{B-A}{d_{m+1}} \quad\left(z \in \mathcal{D}, m \in \mathbb{N}_{k-1}\right)
$$

and

$$
\operatorname{Re}\left\{\frac{f_{m}(z)}{f(z)}\right\} \geq \frac{d_{m+1}}{B-A+d_{m+1}} \quad\left(z \in \mathcal{D}, m \in \mathbb{N}_{k-1}\right) .
$$

The bounds are sharp, with the extremal functions $f_{m+1, \eta}$ defined by (21).

Proof Since

$$
\frac{d_{n+1}}{B-A}>\frac{d_{n}}{B-A}>1 \quad\left(n \in \mathbb{N}_{k}\right)
$$

by Theorem 1 , we have

$$
\sum_{n=k}^{m}\left|a_{n}\right|+\frac{d_{m+1}}{B-A} \sum_{n=m+1}^{\infty}\left|a_{n}\right| \leq \sum_{n=k}^{\infty} \frac{d_{n}}{B-A}\left|a_{n}\right| \leq a_{p} .
$$


Let

$$
g(z)=\frac{d_{m+1}}{B-A}\left\{\frac{f(z)}{f_{m}(z)}-\left(1-\frac{B-A}{d_{m+1}}\right)\right\}=1+\frac{\frac{d_{m+1}}{B-A} \sum_{n=m+1}^{\infty} a_{n} z^{n-p}}{a_{p}+\sum_{n=k}^{m} a_{n} z^{n-p}} \quad(z \in \mathcal{D}) .
$$

Applying (48), we find that

$$
\left|\frac{g(z)-1}{g(z)+1}\right| \leq \frac{\frac{d_{m+1}}{B-A} \sum_{n=m+1}^{\infty}\left|a_{n}\right|}{2 a_{p}-2 \sum_{n=2}^{n}\left|a_{n}\right|-\frac{d_{m+1}}{B-A} \sum_{n=m+1}^{\infty}\left|a_{n}\right|} \leq 1 \quad(z \in \mathcal{D}) .
$$

Thus, we have $\Re[g(z)] \geq 0(z \in \mathcal{D})$ and by (49) we have the assertion (46) of Theorem 9 . Similarly, if we take

$$
h(z)=\left(1+\frac{d_{m+1}}{B-A}\right)\left\{\frac{f_{m}(z)}{f(z)}-\frac{d_{m+1}}{B-A+d_{m+1}}\right\} \quad(z \in \mathcal{D})
$$

and making use of (48), we can deduce that

$$
\left|\frac{h(z)-1}{h(z)+1}\right| \leq \frac{\left(1+\frac{d_{m+1}}{B-A}\right) \sum_{n=m+1}^{\infty}\left|a_{n}\right|}{2 a_{p}-2 \sum_{n=k}^{m}\left|a_{n}\right|-\left(\frac{d_{m+1}}{B-A}-1\right) \sum_{n=m+1}^{\infty}\left|a_{n}\right|} \leq 1 \quad(z \in \mathcal{D}),
$$

which leads us immediately to the assertion (47) of Theorem 9. In order to see that the function $f_{m+1, \eta}$ of the form (21) gives the results sharp, we observe that

$$
\begin{aligned}
& \frac{f_{m+1, \eta}(z)}{\left(f_{m+1, \eta}\right)_{m}(z)}=1-\frac{B-A}{d_{m+1}} \quad\left(z=e^{i \eta}\right), \\
& \frac{\left(f_{m+1, \eta}\right)_{m}(z)}{f_{m+1, \eta}(z)}=\frac{d_{m+1}}{B-A+d_{m+1}} \quad\left(z=e^{i\left(\eta+\frac{\pi}{m-p+1}\right)}\right) .
\end{aligned}
$$

This completes the proof.

Theorem 10 Let the sequence $\left\{d_{n}\right\}$ be increasing and $d_{k}>(m+1)(B-A)$. If $f \in \mathcal{T} \mathcal{W}^{\eta}$, then

$$
\begin{aligned}
& \operatorname{Re}\left\{\frac{f^{\prime}(z)}{f_{m}^{\prime}(z)}\right\} \geq 1-\frac{(m+1)(B-A)}{d_{m+1}} \quad\left(z \in \mathcal{D}, m \in \mathbb{N}_{k-1}\right), \\
& \operatorname{Re}\left\{\frac{f_{m}^{\prime}(z)}{f^{\prime}(z)}\right\} \geq \frac{d_{m+1}}{(m+1)(B-A)+d_{m+1}} \quad\left(z \in \mathcal{D}, m \in \mathbb{N}_{k-1}\right) .
\end{aligned}
$$

The bounds are sharp, with the extremal functions $f_{m+1, \eta}$ defined by (21).

Proof By setting

$$
g(z)=\frac{d_{m+1}}{B-A}\left\{\frac{f^{\prime}(z)}{f_{m}^{\prime}(z)}-\left(1-\frac{(m+1)(B-A)}{d_{m+1}}\right)\right\} \quad(z \in \mathcal{D})
$$

and

$$
h(z)=\left(m+1+\frac{d_{m+1}}{B-A}\right)\left\{\frac{f_{m}^{\prime}(z)}{f^{\prime}(z)}-\frac{d_{m+1}}{(m+1)(B-A)+d_{m+1}}\right\} \quad(z \in \mathcal{D}),
$$

the proof is analogous to that of Theorem 9, and we omit the details. 
Remark 2 By using (17) in Theorems 9 and 10, we obtain the results related to the class $\mathcal{T} \mathcal{W}_{\rho}^{\eta}$.

\section{Concluding remarks}

We conclude this paper by observing that, in view of the subordination relation (9), choosing the functions $\phi$ and $\varphi$, we can consider new and also well-known classes of functions. Let $p>0, n \in \mathbb{N}, x^{n}=1$ and

$$
\mathcal{W}_{\rho}^{n}(p, k ; \varphi ; A, B ; \delta):=\mathcal{W}_{\rho}\left(p, k ; \frac{z \varphi^{\prime}(z)}{p}, \sum_{l=0}^{n-1} \varphi\left(x^{l} z\right) ; A, B ; \delta\right) .
$$

The class $\mathcal{W}_{\rho}^{n}(p, k ; \varphi ; A, B ; \delta)$ generalize well-known classes, which were investigated in earlier works; see, for example, $[5,23,28,30]$. In particular, the class $\mathcal{W}_{\rho}^{n}(p, k ; \varphi ; A, B ; 0)$ contains functions $f \in \mathcal{M}(p, k)$, which satisfies the condition

$$
\frac{z(\varphi * f)^{\prime}(z)}{\sum_{l=0}^{n-1}(\varphi * f)\left(x^{l} z\right)} \prec p \frac{1+A z}{1+B z}
$$

It is related to the class of starlike functions with respect to $n$-symmetric points. Moreover, putting $n=1$, we obtain the class $\mathcal{W}_{\rho}^{1}(p, k ; \varphi ; A, B ; 0)$ defined by the following condition:

$$
\frac{z(\varphi * f)^{\prime}(z)}{(\varphi * f)(z)} \prec p \frac{1+A z}{1+B z}
$$

The class is related to the class of starlike functions. In particular, we have

$$
\mathcal{S}_{p}^{*}(\alpha):=\mathcal{W}_{\rho}^{1}\left(1,2 ; \frac{z^{p}}{1-z} ; 2 \alpha-1,1 ; 0\right)
$$

Analogously, the class

$$
\mathcal{W}_{\rho}^{n}(p, k ; \varphi ; 2 \gamma-p, 1 ; \delta) \quad(0 \leq \gamma<p)
$$

contains functions $f \in \mathcal{M}(p, k)$, which satisfy the condition

$$
\Re\left\{\frac{z(\varphi * f)^{\prime}(z)}{\sum_{l=0}^{n-1}(\varphi * f)\left(x^{l} z\right)}-\gamma\right\}>\delta\left|\frac{z(\varphi * f)^{\prime}(z)}{\sum_{l=0}^{n-1}(\varphi * f)\left(x^{l} z\right)}-p\right| \quad(z \in \mathcal{D}) .
$$

It is related to the class of $\delta$-uniformly convex functions of order $\gamma$ with respect to $n$-symmetric points. Moreover, putting $n=1$, we obtain the class $\mathcal{W}_{n}(p, k ; \varphi ; 2 \gamma-p, 1 ; \delta)$ defined by the following condition:

$$
\Re\left\{\frac{z(\varphi * f)^{\prime}(z)}{(\varphi * f)(z)}-\gamma\right\}>\delta\left|\frac{z(\varphi * f)^{\prime}(z)}{(\varphi * f)(z)}-p\right| \quad(z \in \mathcal{D}) .
$$

The class is related to the class of $\delta$-uniformly convex functions of order $\gamma$. The classes

$$
\begin{aligned}
& \operatorname{UST}(\gamma, \delta):=\mathcal{W}_{0}\left(1,2 ; \frac{z}{1-z} ; 2 \gamma-1,1 ; \delta\right), \\
& \operatorname{UCV}(\gamma, \delta):=\mathcal{W}_{0}\left(1,2 ; \frac{z}{(1-z)^{2}} ; 2 \gamma-1,1 ; \delta\right),
\end{aligned}
$$


are the well-known classes of $\delta$-starlike functions of order $\gamma$ and $\delta$-uniformly convex functions of order $\gamma$, respectively. In particular, the classes UCV $:=\operatorname{UCV}(1,0), \delta-\mathrm{UCV}:=$ $\mathrm{UCV}(\delta, 0)$ were introduced by Goodman [18], and Wisniowska et al. [29] and [19], respectively (see also [20]).

We note that the class

$$
\mathcal{H}_{\mathcal{T}}(\varphi ; \gamma, \delta):=\mathcal{T}^{0}(1,2) \cap \mathcal{W}_{n}(1,2 ; \varphi ; 2 \gamma-1,1 ; \delta)
$$

was introduced and studied by Raina and Bansal [24].

If we set

$$
h\left(\alpha_{1}, z\right):=z_{q} F_{s}\left(\alpha_{1}, \ldots, \alpha_{q} ; \beta_{1}, \ldots, \beta_{s} ; z\right),
$$

where ${ }_{q} F_{s}$ is the generalized hypergeometric function, then we obtain the class

$$
\mathcal{U H}(q, s, \lambda, \gamma, \delta):=\mathcal{H}_{\mathcal{T}}\left(\lambda h\left(\alpha_{1}+1, z\right)+(1-\lambda) h\left(\alpha_{1}, z\right) ; \gamma, \delta\right) \quad(0 \leq \lambda \leq 1)
$$

defined by Srivastava et al. [26].

Let $\lambda$ be a convex parameter. A function $f \in \mathcal{M}(p, k)$ belongs to the class

$$
\mathcal{V}_{\lambda}(\varphi ; A, B):=\mathcal{W}\left(\lambda \frac{\varphi(z)}{z}+(1-\lambda) \varphi^{\prime}(z), z ; A, B ; 0\right)
$$

if it satisfies the following condition:

$$
\lambda \frac{(\varphi * f)(z)}{z}+(1-\lambda)(\varphi * f)^{\prime}(z) \prec \frac{1+A z}{1+B z} .
$$

Moreover, a function $f \in \mathcal{M}(p, k)$ belongs to the class

$$
\mathcal{D}_{\lambda}(\varphi ; A, B):=\mathcal{W}\left(\lambda \frac{\varphi(z)}{z}+(1-\lambda) \varphi^{\prime}(z) ; A, B ; 0\right)
$$

if it satisfies the following condition:

$$
\frac{z(\varphi * f)^{\prime}(z)+(1-\lambda) z^{2}(\varphi * f)^{\prime \prime}(z)}{\lambda(\varphi * f)(z)+(1-\lambda) z(\varphi * f)^{\prime}(z)} \prec \frac{1+A z}{1+B z} .
$$

The considered classes are defined by using the convolution $\varphi * f$ or equivalently by the linear operator

$$
J_{\varphi}: \mathcal{M}(p, k) \rightarrow \mathcal{M}(p, k), \quad J_{\varphi}(f)=\varphi * f .
$$

By choosing the function $\varphi$, we can obtain a lot of important linear operators, and in consequence new and also well-known classes of functions. We can listed here some of these linear operators as the Salagean operator, the Cho-Kim-Srivastava operator, the DziokRaina operator, the Hohlov operator, the Dziok-Srivastava operator, the Carlson-Shaffer operator, the Ruscheweyh derivative operator, the generalized Bernardi-Libera-Livingston 
operator, the fractional derivative operator and so on (see, for the precise relationships $[14,17])$.

If we apply the results presented in the paper to the classes discussed above, we can lead to several results. Some of these were obtained in earlier works; see, for example, [3-17, 21, 23-26, 30-35].

\section{Competing interests}

The author declares that they have no competing interests.

\section{Acknowledgements}

Dedicated to Professor Hari M Srivastava.

Received: 20 December 2012 Accepted: 18 March 2013 Published: 5 April 2013

\section{References}

1. Montel, P: Leçons sur les Fonctions Univalentes ou Multivalentes. Gauthier-Villars, Paris (1933)

2. Silverman, $\mathrm{H}$ : Univalent functions with varying arguments. Houst. J. Math. 7, 283-287 (1981)

3. Srivastava, HM, Owa, S: Certain classes of analytic functions with varying arguments. J. Math. Anal. Appl. 136, 217-228 (1988)

4. Aouf, MK, Dziok, J: Distortion and convolutional theorems for operators of generalized fractional calculus involving Wright function. J. Appl. Anal. 14(2), 183-192 (2008)

5. Aouf, MK, Hossen, HM, Srivastava, HM: Some families of multivalent functions. Comput. Math. Appl. 39, 39-48 (2000)

6. Aouf, MK, Srivastava, HM: Some families of starlike functions with negative coefficients. J. Math. Anal. Appl. 203(3), 762-790 (1996)

7. Chen, M-P, Irmak, H, Srivastava, HM: Some families of multivalently analytic functions with negative coefficients. J. Math. Anal. Appl. 214(2), 674-690 (1997)

8. Cho, NE, Bulboaca, T, Srivastava, HM: A general family of integral operators and associated subordination and superordination properties of some special analytic function classes. Appl. Math. Comput. 219, 2278-2288 (2012)

9. Cho, NE, Kim, IH, Srivastava, HM: Sandwich-type theorems for multivalent functions associated with the Srivastava-Attiya operator. Appl. Math. Comput. 217, 918-928 (2010)

10. Dziok, J: A new class of multivalent analytic functions defined by the Hadamard product. Demonstr. Math. 44(2), 233-251 (2011)

11. Dziok, J: Classes of functions defined by certain differential-integral operators. J. Comput. Appl. Math. 105(1-2), 245-255 (1999)

12. Dziok, J: Applications of the Jack lemma. Acta Math. Hung. 105, 93-102 (2004)

13. Dziok, J: On the convex combination of the Dziok-Srivastava operator. Appl. Math. Comput. 188(2), 1214-1220 (2007)

14. Dziok, J, Raina, RK: Families of analytic functions associated with the Wright generalized hypergeometric function. Demonstr. Math. 37(3), 533-542 (2004)

15. Dziok, J, Raina, RK, Srivastava, HM: Some classes of analytic functions associated with operators on Hilbert space involving Wright's generalized hypergeometric function. Proc. Jangieon Math. Soc. 7, 43-55 (2004)

16. Dziok, J, Srivastava, HM: Certain subclasses of analytic functions associated with the generalized hypergeometric function. Integral Transforms Spec. Funct. 14, 7-18 (2003)

17. Dziok, J, Srivastava, HM: Classes of analytic functions associated with the generalized hypergeometric function. Appl. Math. Comput. 103, 1-13 (1999)

18. Goodman, AW: On uniformly convex functions. Ann. Pol. Math. 56, $87-92$ (1991)

19. Kanas, S, Wisniowska, A: Conic regions and k-uniform convexity. J. Comput. Appl. Math. 105, 327-336 (1999)

20. Kanas, S, Srivastava, HM: Linear operators associated with k-uniformly convex functions. Integral Transforms Spec. Funct. 9, 121-132 (2000)

21. Kulkarni, SR, Naik, UH, Srivastava, HM: An application of fractional calculus to a new class of multivalent functions with negative coefficients. Comput. Math. Appl. 38(5-6), 169-182 (1999)

22. Littlewood, JE: On inequalities in theory of functions. Proc. Lond. Math. Soc. 23, 481-519 (1925)

23. Liu, J-L, Srivastava, HM: Certain properties of the Dziok-Srivastava operator. Appl. Math. Comput. 159, 485-493 (2004)

24. Raina, RK, Bansal, D: Some properties of a new class of analytic functions defined in terms of a Hadamard product. J. Inequal. Pure Appl. Math. 9, Article ID 22 (2008)

25. Raina, RK, Srivastava, HM: A unified presentation of certain subclasses of prestarlike functions with negative coefficients. Comput. Math. Appl. 38(11-12), 71-78 (1999)

26. Ramachandran, C, Shanmugam, TN, Srivastava, HM, Swaminathan, A: A unified class of $k$-uniformly convex functions defined by the Dziok-Srivastava linear operator. Appl. Math. Comput. 190, 1627-1636 (2007)

27. Silvia, EM: Partial sums of convex functions of order $\alpha$. Houst. J. Math. 11, 397-404 (1985)

28. Sokół, J: On some applications of the Dziok-Srivastava operator. Appl. Math. Comput. 201, 774-780 (2008)

29. Sokół, J, Wiśniowska, A: On some classes of starlike functions related with parabola. Folia Sci. Univ. Tech. Resov. 121, 35-42 (1993)

30. Srivastava, HM, Murugusundaramoorthy, G, Sivasubramanian, S: Hypergeometric functions in the parabolic starlike and uniformly convex domains. Integral Transforms Spec. Funct. 18, 511-520 (2007)

31. Wang, Z-G, Li, Q-G, Jiang, Y-P: Certain subclasses of multivalent analytic functions involving the generalized Srivastava-Attiya operator. Integral Transforms Spec. Funct. 21, 221-234 (2010)

32. Wang, Z-G, Liu, Z-H, Xiang, R-G: Some criteria for meromorphic multivalent starlike functions. Appl. Math. Comput. $218,1107-1111(2011)$ 
33. Wang, Z-G, Liu, Z-H, Cataş, A: On neighborhoods and partial sums of certain meromorphic multivalent functions. Appl. Math. Lett. 24(6), 864-868 (2011)

34. Wang, Z-G, Sun, Y, Xu, N: Some properties of certain meromorphic close-to-convex functions. Appl. Math. Lett. 25 454-460 (2012)

35. Wang, Z-G, Sun, Y, Zhang, Z-H: Certain classes of meromorphic multivalent functions. Comput. Math. Appl. 58, 1408-1417 (2009)

36. Wilf, HS: Subordinating factor sequence for convex maps of the unit circle. Proc. Am. Math. Soc. 12, 689-693 (1961)

doi:10.1186/1687-1812-2013-86

Cite this article as: Dziok: Classes of multivalent analytic and meromorphic functions with two fixed points. Fixed Point Theory and Applications 2013 2013:86.

Submit your manuscript to a SpringerOpen ${ }^{\odot}$ journal and benefit from:

- Convenient online submission

- Rigorous peer review

- Immediate publication on acceptance

- Open access: articles freely available online

- High visibility within the field

- Retaining the copyright to your article

Submit your next manuscript at $>$ springeropen.com 